\title{
Evolution of Massive Stars at Low Metallicity
}

\author{
Georges Meynet ${ }^{1}$, Nolan R. Walborn ${ }^{2}$, Ian Hunter ${ }^{3}$, Christophe \\ Martayan $^{4}$, Allard Jan van Marle ${ }^{5}$, Sergey Marchenko ${ }^{6}$, Jorick S. \\ Vink $^{7}$, Marco Limongi ${ }^{8}$, Emily M. Levesque ${ }^{9}$, and Maryam Modjaz ${ }^{10}$ \\ ${ }^{1}$ Observatory of Geneva University, Switzerland \\ email: georges.meynet@obs.unige.ch \\ ${ }^{2}$ Space Telescope Science Institute, USA \\ email: walborn@stsci.edu \\ ${ }^{3}$ Queen's University of Belfast, UK \\ email: i.hunter@qub.ac.uk \\ ${ }^{4}$ Observatoire Royal de Belgique, Belgium, and, Observatoire de Paris-Meudon, France \\ email: martayan@oma.be \\ ${ }^{5}$ Bartol Research Institute, University of Delaware, USA \\ email: marle@udel.edu \\ ${ }^{6}$ Western Kentucky University, USA \\ email: sergey .marchenko@wku.edu \\ ${ }^{7}$ Armagh Observatory, UK \\ email: jsv@arm.ac.uk \\ ${ }^{8}$ Istituto Nazionale di Astrofisica, Italy, and Monash University, Australia \\ email: marco@oa-roma.inaf.it \\ ${ }^{9}$ University of Hawaii, USA \\ email: emsque@IfA. Hawaii.Edu \\ ${ }^{10}$ UC Berkeley Astronomy Department, USA \\ email: mmodjaz@astro.berkeley.edu
}

\begin{abstract}
This paper reports the contributions made on the occasion of the Special Session entitled "Evolution of Massive Stars at Low Metallicity" which was held on Sunday, December 9, 2007 in Kauai (USA).
\end{abstract}

Keywords. stars: abundances, early-type, evolution, mass loss, emission-line, Be, rotation; gamma rays: bursts

\section{Introduction}

Georges Meynet

Understanding the evolution of massive stars at low and very low metallicity is a requirement to address questions such as the nature of the sources of the reionization in the early Universe, the evolution of the interstellar abundances during the early phases of the evolution of galaxies, for finding possible signatures of primordial stellar populations in the integrated light of very distant galaxies and for discovering which objects are the progenitors of the long soft Gamma Ray Bursts. At present, the most "iron" poor objects known in the Universe are not very far from us since they are galactic halo stars. Provided these stars are trustworthy very metal poor stars (a view recently challenged by Venn \& Lambert 2008), these objects offer a unique opportunity to study the yields of the first generations of stars and supernovae. Very interestingly, many observed features 
are unexpected and will probably require some revision of the classical ideas on how very metal poor massive stars evolve. To cite a few of them, let us recall that in the abundance patterns of the most metal poor halo stars, 1) no sign of Pair Instability Supernovae has been observed (Cayrel et al. 2004), 2) a high plateau of the $\mathrm{N} / \mathrm{O}$ ratios (as a function of $\mathrm{O} / \mathrm{H}$ ) are obtained requiring the activity of efficient sources of primary nitrogen (see e.g. Spite et al. 2005), 3) simultaneously the $\mathrm{C} / \mathrm{O}$ ratio as a function of $\mathrm{O} / \mathrm{H}$ shows an upturn at a metallicity of $[\mathrm{O} / \mathrm{H}] \sim-2$ (Spite et al. 2005, see also Pettini, this volume), 4) a significant fraction of very metal poor stars are C-rich stars showing very peculiar abundance pattern at their surface (Beers \& Christlieb 2005), 5) very helium-rich stars and 6) stars with high abundance of sodium and low abundance of oxygen are detected in globular clusters (Gratton et al. 2004; Piotto et al. 2005). In order to go further in the understanding of these very interesting questions, we have to obtain detailed and solid observations of massive stars at low metallicity in our neighborhood, produce models able to reproduce these observed features and then, using the same successful physics, explore the consequences for primordial and very metal poor stars.

The contributions presented during this special session brought new and very interesting results in the following areas of research: a first series of three contributions presented recent observations of massive stars at low metallicity, a second series of three contributions discussed the important question of the variation of the stellar winds with metallicity, then Pop III interior models have been presented and finally two presentations were devoted to the study of Gamma Ray Burst hosts.

\section{The Onfp Class in the Magellanic Clouds}

Nolan R. Walborn

The primary defining characteristic of Onfp spectra is the presence of an absorption reversal in the He II $\lambda 4686$ emission line. Most members of the class also have substantially broadened absorption and emission lines. These properties suggest rapid rotation and perhaps disk structures - but see Bouret, Hillier, \& Lanz, this volume, who reproduce this $\lambda 4686$ profile with just wind clumping and rotation. The Galactic prototypes are the two brightest Of stars in the sky, $\zeta$ Puppis and $\lambda$ Cephei. Luminosity classification of these objects is problematic, since the line profile of the primary He II criterion is peculiar. Recent spectroscopic surveys of OB stars in the Magellanic Clouds have found increasing numbers of the Onfp class, currently numbering about two dozen. Their spectral types span the entire O-type range, while their reliable absolute magnitudes range between those of classes V and I. Significant spectral variations are seen in all objects with multiple observations, including single-peaked $\lambda 4686$ profiles, and two of them have been found to be spectroscopic binaries. Most of them are located near the peripheries of clusters or associations, with a few exceptions in compact clusters. The Onfp linebroadening distribution is distinct and shifted toward larger values from those of normal O dwarfs and supergiants with $>99.99 \%$ confidence (analysis by Ian Howarth). These objects are of interest in the contexts of massive star formation, binary mergers, and GRB progenitors, the last particularly at the SMC metallicity.

\section{Rotational Mixing in Magellanic Cloud Massive Stars} Ian Hunter

The prediction that core processed material is rotationally mixed into the photosphere is now considered a fundamental property in evolutionary models of massive stars. However, 
such theories have never been observationally tested, for example, one would expect the fastest rotators to be the most mixed but analyses of such fast rotators have never before been carried out. The analysis of over 100 LMC B-type massive stars has been carried out and surface nitrogen enrichments are used as an indicator of the efficiency of the mixing mechanism. Models including rotation have been generated to best fit the observed data and while the models can reproduce a significant fraction of the data there are a number of discrepancies between these theoretical models and the observations. In particular several fast rotators with little evidence of rotational mixing are observed despite being close to the end of their hydrogen burning lifetimes. Additionally both populations of mainsequence slow rotators and evolved blue supergiants with significant chemical enrichments are seen. These populations violate the current theories of rotational mixing and challenge our understanding of massive star evolution.

\section{The Low Metallicity Effects on the B and Be Stars}

Christophe Martayan

We present new observational results of the low metallicity effects on B and Be star populations of the Magellanic Clouds (MC). First, we show the results obtained with the VLT-GIRAFFE about the rotational velocities during the main sequence and at the ZAMS. These results indicate that the lower the metallicity, the higher the rotational velocities of B and Be stars. The ZAMS rotational velocities for Be stars we found are mass and metallicity-dependent. Second, we present new clues on the appearance of Be stars and explain the difference of evolution for the more massive of them between the Milky Way (MW) and the Small Magellanic cloud (SMC). Third, with a cross-matching between the 520 B-type stars observed with the VLT and the large photometric survey MACHO, we report on the detection of binaries and for the first time of short-term multi-periodicity in $\sim 30$ Be stars and $\sim 10$ B stars in the SMC. This result plaids in favor of pulsations at low metallicity, which was not foreseen by theory. Fourth, thanks to a $\mathrm{H} \alpha$ survey conducted with the ESO-WFI in slitless spectroscopic mode, we obtained 8 millions of spectra in the MC, and we found that the proportion of Be stars to B stars in $85 \mathrm{SMC}$ open clusters is higher than in the MW.

\section{Continuum Driven Winds from Super-Eddington Massive Stars}

Allard Jan van Marle

If a star exceeds the Eddington limit, continuum scattering becomes a very efficient mechanism for removing mass from a star, producing mass loss rates that are several orders of magnitude larger than those achieved by line driving. This, coupled to the fact that continuum driving does not depend on the metallicity of the star makes it particularly well suited to removing mass from zero- and low-metallicity stars, which is necessary to prevent them from exploding as pair instability supernovae. Numerical simulations using the porosity length formalism derived by Owocki et al. (2004), show that super-Eddington stars can drive steady winds. However, if the mass loss rate becomes so large that the mechanical luminosity of the wind exceeds the radiative luminosity of the star, then the wind becomes unstable as part of the material will fall back toward the star. See van Marle et al. (2008). 


\section{Wind-Clumping in Low-Z Environment: Theory and Observations}

Sergey Marchenko

We briefly review theoretical expectations for the wind-clumping factors in the environments with a low heavy-metal content. We show that, based on the recent observations of a small sample of Population I Wolf-Rayet stars in the Small Magellanic Cloud, general properties of the detected wind-clumps closely match the corresponding characteristics of small-scale inhomogeneities in the winds of Galactic Wolf-Rayet stars.

\section{Mass Loss at Very Low Z}

\section{Jorick Vink}

Stellar wind models for $\mathrm{OB}$ and WR stars as a function of $\mathrm{Z}$ are presented. Since the mass-loss rate is determined by the Fe lines in the inner wind, the theoretical dependence is found to be relatively steep, with $\dot{M} \propto Z^{0.7-0.8}$ down to $Z=10^{-2} Z_{\odot}$ (Vink et al. 2001, Vink \& de Koter 2005). The new prediction of Z-dependent mass-loss for WR winds may be particularly relevant for the progenitor evolution of long duration gamma-ray bursts (GRBs), which are widely believed to be rapidly rotating WR stars. Testing the rotational properties and asymmetries of WR stars at low $Z$ using recent spectropolarimetric data, Vink (2007) discussed the threshold metallicity for making a long-duration GRB.

Turning attention to the early Universe at extremely low $Z$, we emphasize a flattening of the $\dot{M}-Z$ dependence below $Z=10^{-3} Z \odot$ for late type WC stars. This might suggest that mass loss could still play a relevant role for massive stars in the early Universe, especially if these stars turn out to be close to their Eddington limit, e.g. undergoing LBV outbursts (Vink \& de Koter 2005). It has also been suggested that characteristic LBV S Doradus mass-loss behavior in the present-day Universe might indicate that some LBVs could be direct precursors of supernovae (Kotak \& Vink 2006).

\section{Pop III Stellar Models}

\section{Marco Limongi}

We presented our latest set of massive star models with initial zero metallicity (in the mass range 13-80 solar masses) and their associated explosive yields. The presupernova models have been computed by means of the FRANEC code (050419) which is described in Limongi \& Chieffi (2006). The explosive yields are computed by means of a hydro code with an induced explosion in the framework of the kinetic bomb. First, we described the main differences in the evolutionary properties between zero and solar metallicity models. Among these, the most relevant one being the substantial primary ${ }^{14} \mathrm{~N}$ production in zero metallicity models. This production is due to the fact that protons, in the $\mathrm{H}$ shell tail, are ingested by the underlying He convective shell. Such an ingestion activates a hot CNO cycle leading to a substantial production of primary ${ }^{14} \mathrm{~N}$. Such a partial mixing between the He convective shell and the overlying H-rich zone is a rather common occurrence in stellar models of initial zero metallicity (see e.g. Woosley \& Weaver 1982) because of the low-entropy barrier that develops at the H-He interface in these stars. The second difference between solar and zero metallicity models is the well known odd-even effect, i.e., the difference between the production factors of the odd (Na-Sc) and the even (Ne$\mathrm{Ca})$ nuclei is much larger in the zero metallicity models compared to their corresponding solar metallicity ones. Finally, we presented a comparison between the explosive yields obtained for the zero metallicity models and the element abundance ratios observed in 
extremely metal poor stars (EMPS). These stars, in fact, probably formed in the very early epochs of Galaxy formation by gas clouds chemically enriched by the first stellar generations (POP III). Hence, whether or not they are associated to single supernovae or single burst events, they provide very useful constraints to test presupernova models, supernova explosion and nucleosynthesis theories as well as they can be used to infer the nature of the first generations of stars and supernovae. We showed that the element abundance pattern of both the NORMAL and the C-RICH EMPS can be explained in terms of enrichment of a population of STANDARD POP III core collapse supernovae (CC-SNe) with fluctuations of the IMF. In particular we showed that clouds dominated by the ejecta of high mass $\mathrm{SNe}$ are characterized by a chemical composition typical of the C-RICH EMPS, while clouds dominated by the ejecta of the lower mass SNe are characterized by a composition typical of the NORMAL EMPS.

\section{Metal-Poor Galaxies and Gamma-Ray Burst Hosts}

Emily Levesque

We have used the newest generation of the Starburst99/Mappings code to generate an extensive suite of models covering a wide range of physical parameters suitable for metalpoor galaxies and gamma-ray burst hosts. We use our theoretical models with optical emission line diagnostic diagrams to constrain the star-formation histories and ISM properties of metal-poor galaxies and gamma-ray burst hosts. Our comparisons reveal several important short-comings of the current model atmospheres and stellar evolutionary tracks in metal-poor environments - for more discussion, see Levesque et al. (2008, in prep). We have applied our diagnostics to the host galaxy of GRB 060505, one of the most hotly contested GRBs to date. We do not find compelling evidence to suggest that GRB 060505 originated in a long-duration core collapse progenitor. Our emission line diagnostic analysis suggests that the environment of GRB 060505 is more consistent with the host environments of compact-object-merger GRB progenitors (Levesque \& Kewley 2007). In the future, a detailed understanding of stellar evolution at low metallicities will provide a deeper understanding of the unique and unusual environments of GRB progenitors.

\section{Metallicities at the Sites of Nearby SN Ic}

\section{Modjaz}

We show clues as to the production conditions of long-duration Gamma-ray Bursts (GRBs) by comparing the chemical abundances at the sites of five broad-lined SN Ic that accompany nearby GRBs with those of twelve nearby broad-lined SN Ic with no observed GRBs. We show that the oxygen abundances at the GRB sites are significantly lower than those found near ordinary broad-lined SN Ic. We demonstrate that neither SN selection effects (SN found via targeted vs. non-targeted surveys) nor the choice of the three strong-line metallicity diagnostic are convincing causes of the observed trend. Though the sample size is small, the observations are consistent with the hypothesis that low metal abundance is the main cause of some massive stars becoming SN-GRB, with a cut-off metallicity of $0.2-0.6 \mathrm{Z}_{\odot}$, depending on the adopted metallicity scale and solar abundance value (Modjaz et al. 2008). 


\section{References}

Beers, T. C. \& Christlieb, N. 2005, ARA\&A, 43, 531

Cayrel, R., Depagne, E., \& Spite, M. 2004, A\&A 416, 1117

Chieffi, A. \& Limongi, M. 2006, ApJ, 647, 483

Gratton, R., Sneden, C., \& Carretta, E. 2004, ARA\&A, 42, 385

Kotak, R. \& Vink, J. S. 2006, A\& A, 460, L5

Levesque, E. M. \& Kewley, L. J. 2007, ApJ, 667, L121

Modjaz, M., Kewley, L., Kirshner, R. P. et al. 2008, AJ, 135, 1136

Owocki, S. P., Gayley, K. G., \& Shaviv, N. J. 2004, ApJ, 616, 525

Piotto, G., Villanova, S. \& Bedin, L. R. 2005, ApJ, 621, 777

Spite, M., Cayrel, R., \& Plez, B. 2005, A\& A, 430, 655

van Marle, A. J., Owocki, S. P., \& Shaviv, N. J. 2008, in: B. O'Shea, A. Heger \& T. Abel (eds.), First Stars III (New York: AIP), in press (arXiv:0708.4207)

Venn, K. A. \& Lambert, D. L. 2008, ApJ, in press, (arXiv:0801.0752)

Vink, J. S., 2007, A\& A 469, 707

Vink, J. S., \& de Koter, A. 2005, A\& A 442, 587

Vink, J. S., de Koter, A., \& Lamers, H. J. G. L. M. 2001, A\&A 369, 574

Woosley, S. E. \& Weaver, T. A., 1982, in: M.J. Rees \& R.J. Stoneham (eds.), Supernovae: A Survey of Current Research, (Dordrecht: Reidel), 79 